\title{
Experimental Study on Influence of Fuel Injection Pressure on Dynamic Flow Characteristics of Solenoid Valve Injector
}

\author{
Janwen $\mathrm{Li}^{1}$, a , Wei $\mathrm{Lei}^{2, \mathrm{~b}}$, Yanhao $\mathrm{Li}^{1, \mathrm{c}}$ and Bin $\mathrm{Liu}^{1, \mathrm{~d}}$ \\ ${ }^{1}$ School of Electrical and Electronic Engineering, Tianjin University of Technology, Tianjin 300161, \\ China; \\ ${ }^{2}$ School of Postgraduate Training Brigade, Military Transportation University, Tianjin 300161, China. \\ ajianwen_li@sina.com, blwssg182@163.com, c497762003@qq.com, d39510893@qq.com
}

Keywords: Dynamic flow; electronically controlled injector; gasoline engine; dynamic flow range.

\begin{abstract}
The factors influencing the dynamic flow characteristics of injectors are discussed, and a set of experimental system for measuring dynamic flow is designed and developed. Taking a certain fuel injector produced by Wenzhou manufacturer as the research object, the circulating fuel injection quantity of the injector under different pulse widths was measured. The dynamic flow characteristic curve was obtained by fitting the polynomial of MATLAB to calculate the dynamic flow of the injector range. The influence of injection pressure on the dynamic flow characteristics of injectors was analyzed, which provided a reference for ECU to precisely control injector injection quantity.
\end{abstract}

\section{Introduction}

Injector, as the executive unit of electronically controlled fuel injection system of gasoline engine, its working characteristics, flow characteristics and spray characteristics have a direct impact on the performance of the engine such as emission, fuel economy and dynamic performance [1]. The linearity and linear range of the flow characteristics curve of the electronic fuel injector is an important parameter to evaluate the injector quality. Factors that influence the linearity and linearity range of the injector flow rate characteristic in addition to the inherent configuration of the injector, there is also the injection pressure and the drive voltage of the drive module. In the actual working process of the fuel injector, the working conditions of the gasoline engine are constantly changing, and the fuel injection pressure of the fuel tank is not always constant. As the injection pressure changes, the injector fuel injection cycle also changes, in order to further study the injection pressure on the injector flow characteristics of the law, this paper designed a set of test system for measuring dynamic flow characteristics, Taking a fuel injector produced by a certain company in Wenzhou as the test object and using the polynomial fitting of MATLAB to obtain the dynamic flow characteristic curve and studying the influence law of injection pressure on the dynamic flow characteristics of the injector [2].It provides a reference for ECU to precisely control fuel injection quantity of injector.

\section{Test System Construction}

In this paper, a device is designed to measure the injector flow characteristics, including the measurement of static flow and dynamic flow. The device mainly consists of the gas circuit section, the oil circuit section and the electronically controlled section. Gas circuit part includes air pump, pressure gauge, pneumatic switch, pneumatic cylinder and pressure tank, mainly for the entire test system to provide pressure sources; part of the oil tank, pump, into the oil rail, flow meter, take the liquid chamber, fuel injection As well as the oil return rail, mainly for the test system to provide the test medium at the same time the amount of fuel injection injector; electronic control part, including PLC, counter, electromagnetic proportional valve, single solenoid valve, two two-way solenoid valve and Pressure sensor, the main control of gas and oil off, provide the drive pulse for the injector. System diagram shown in Figure 1. 


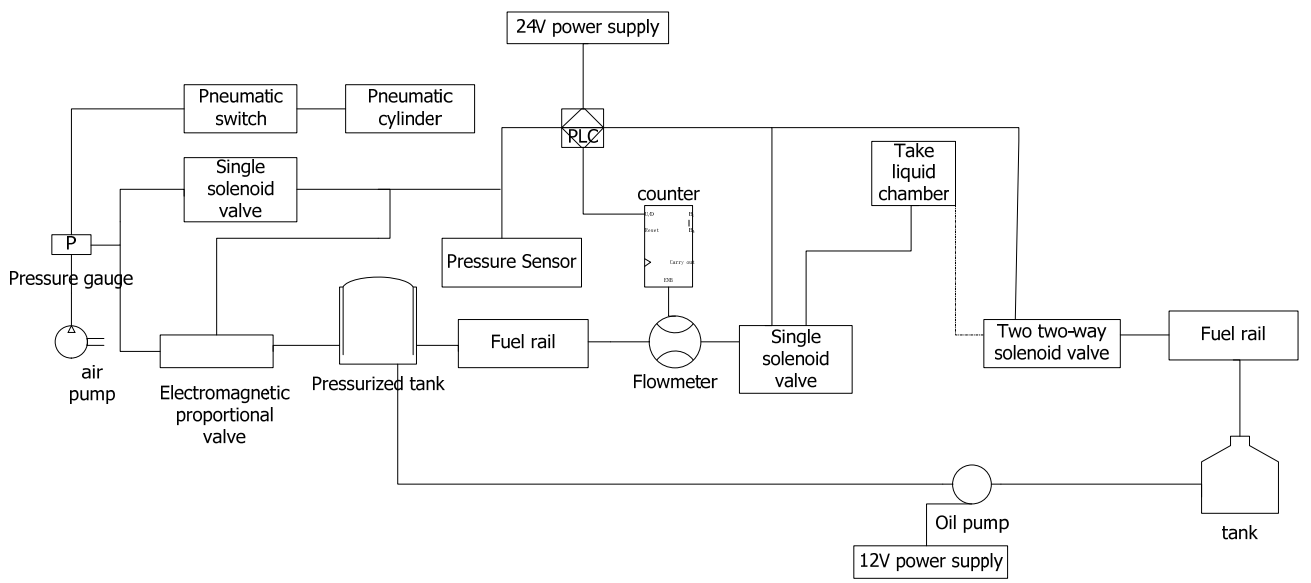

Figure 1. Fuel injector dynamic flow test system composition diagram

\section{Expert Mental Design}

To obtain the complete dynamic flow characteristic curve, it is very important to have high resolution in testing injector non-linearity [3]. In the range of the injection pulse width that requires enhanced resolution, the injector flow should be measured in increments of $0.1 \mathrm{mms}$ pulse width. Other fuel injection pulse width range should be $1 \mathrm{~ms}$ pulse width measurement injector flow.

Since the fuel injector single injection is very small, each sampling point for multiple injection and then average. Experiment, the test cycle is $12 \mathrm{~ms}$, the injector drive circuit selection PLC as the control unit, in which the working voltage of PLC is $24 \mathrm{~V}$, the driving voltage is $12 \mathrm{~V}$, the injector fuel inlet hydraulic control at $250 \mathrm{kPa} 0.2 \mathrm{kPa}$, the experimental medium using aviation kerosene. The injection signal generated by the driving circuit is transmitted to the injector for controlling the injector to be turned on and off. The injection period and the injection pulse width are set by the host computer. The flow of the injector is measured by the flow meter, Specific experimental program is as follows:

(1) To complete the pulse width of $5 \mathrm{~ms}$, the number of shots for the 500 pre-injection.

(2) The injection pulse width gradually increases from 0 to $10 \mathrm{~ms}$, and the increment is $0.1 \mathrm{~ms}$ in the pulse width range of $0 \sim 2 \mathrm{~ms}$; the increment is $1 \mathrm{~ms}$ in the pulse width range of $2 \sim 9 \mathrm{~ms}$; in the pulse of $9 \sim 10 \mathrm{~ms}$ Wide range, increments $0.2 \mathrm{~ms}$. The number of shots per sampling point is 2000 times.

(3) Under the driving voltage of $12 \mathrm{~V}$, repeat the step (2) to measure the injector flow characteristics of the oil pressure of $200 \mathrm{kPa}, 300 \mathrm{kPa}, 350 \mathrm{kPa}$ and $400 \mathrm{kPa}$.

Oil pressure of $200 \mathrm{kPa}, 250 \mathrm{kPa}, 300 \mathrm{kPa}, 350 \mathrm{kPa}$ and $400 \mathrm{kPa}$ test conditions were tested five groups, and then averaged to reduce the impact of accidental error caused by a single test.

\section{Test Data Processing and Analysis}

This selection of flow meter GICAR series of micro-flow meter is a turbine principle designed to measure the flow of special micro-meter, it has a very high accuracy. In the test of injector flow, the flow meter turbine by the counter to obtain the number of open, as the data output, the instrument coefficient of 0.6 , so the injector single injection quantity is calculated as follows:

$$
Q=\frac{n \times 0.6}{2000}
$$

Where: $\mathrm{Q}$ is the amount of circulating fuel injection; $\mathrm{N}$ is the number of times.

Through the above formula, calculate the fuel injection pulse width of each cycle of fuel injection, the use of polynomial fitting MATLAB derived 12V drive voltage, 250 kappa injection pressure dynamic flow characteristics, as shown in Figure 2: 


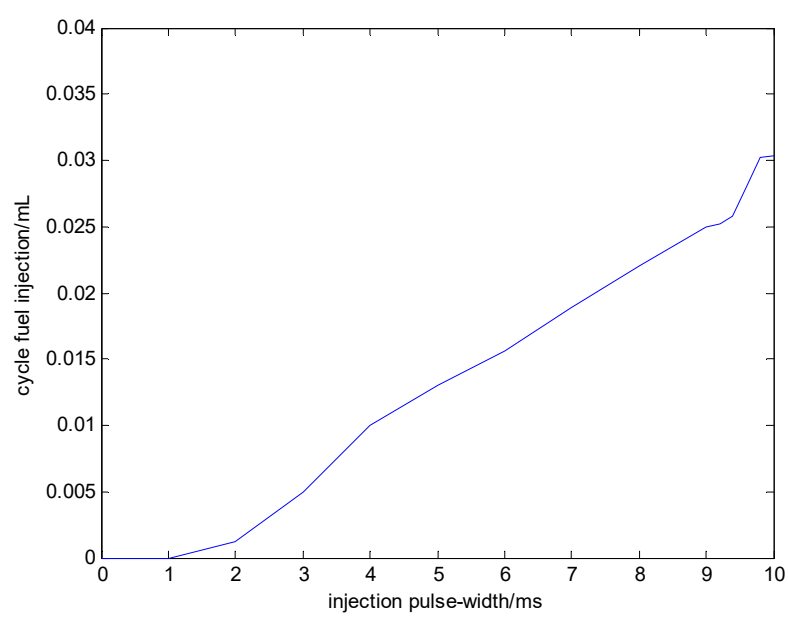

Figure 2. Dynamic Flow Curve at 250 kappa

\subsection{Dynamic Flow Characteristics Analysis.}

It can be seen from Fig. 2 that in the injection pulse width range of $4 \sim 9 \mathrm{~ms}$, the fuel injection quantity of the injector exhibits a good linear relationship with the change of the injection pulse width, which can meet the normal pulse width of the injector. The request. The curve corresponding to the $4 \sim 9 \mathrm{~ms}$ time is linearly fitted by the least-squares method. The resulting straight line is shown in Figure 3, and the fitted linear equation is:

$$
y=0.003 * x-0.0021
$$

Where: $\mathrm{y}$ is the circulation injection quantity; $\mathrm{x}$ is the injection pulse width.

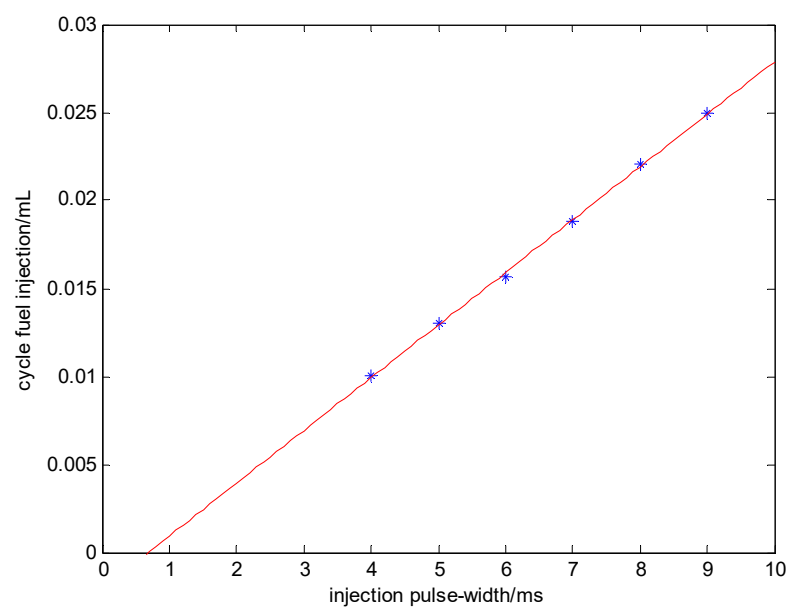

Figure 3. Dynamic flow characteristics fit a straight line

The static flow of the injector can be approximated by $\mathrm{mL} \cdot \mathrm{ms}-1$ from the fitted straight line, but the injection flow performance of the injector is generally used in the dynamic flow range [4]. Injectors at their respective pulse widths, the maximum linear flow point is the maximum available dynamic flow qmax, the smallest linear flow point is the minimum available dynamic flow qmin, and the expression of the dynamic flow range can be derived from the definition of the dynamic flow range. The following formula:

$$
D F R=\frac{q_{\max }}{q_{\min }}
$$

\subsection{Effect of Injection Pressure on Dynamic Flow Characteristics.}

When testing, the drive voltage is maintained at $12 \mathrm{~V}$, adjust the supply pressure on the control panel to change the injection pressure of the injector, were measured when the injection pressure of $200 \mathrm{kPa}, 250 \mathrm{kPa}, 300 \mathrm{kPa}, 350 \mathrm{kPa}$ and $400 \mathrm{kPa}$ when the dynamic flow curve, as shown in Figure 4. 


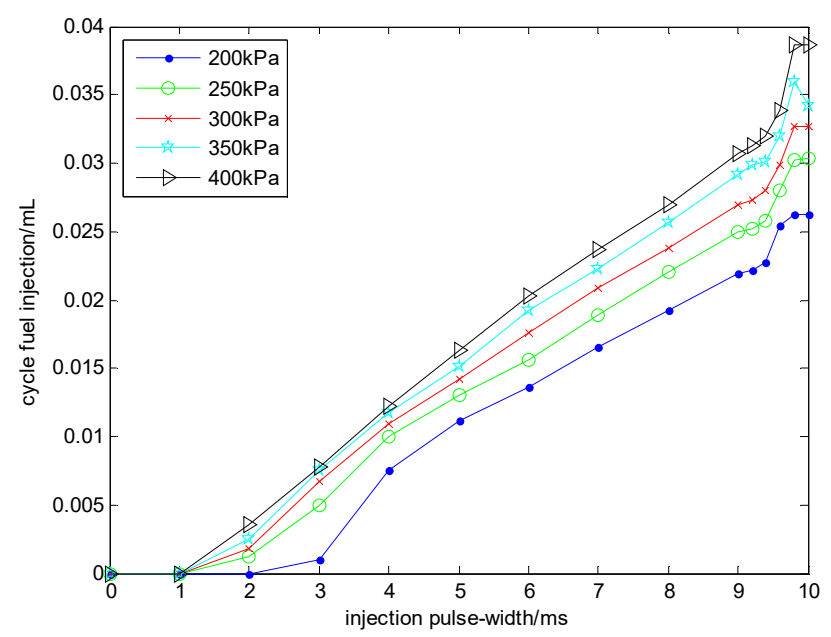

Figure 4. Dynamic flow curve under different injection pressure

From the above analysis, it can be seen that the injection pressure has little effect on the dynamic flow characteristics during the pulse injection stage of small fuel injection; with the increase of the injection pulse width, especially in the linear range of injectors, fuel injection The quantity is proportional to the injection pressure, indicating that the injection pressure mainly affects the linear section of the injector, and the greater the injection pressure, the higher the amount of fuel per unit of time; by comparing the linear range under each injection pressure, spray The linear range of the oiler is basically the same; Comparing the dynamic flow range under various pressure conditions, the injector's dynamic flow range is the largest when the injection pressure is $300 \mathrm{kPa}$, and the injector's dynamic flow range tends to be stable when the injection pressure is more than $300 \mathrm{kPa}$.

\section{Summary}

1) The influence of injection pressure on the dynamic flow characteristics of injector mainly reflected in the linear section, the greater the injection pressure, the higher the amount of fuel injection per unit time.

2) The influence of injection pressure on the dynamic flow curve shape is very small. The linear section of the dynamic flow curve fluctuates with a small injection pressure.

3) Injector dynamic flow range with the injection pressure changes, but there is no obvious linear relationship.

\section{References}

[1]. JH Li, DW Liu, GW Shi, et al. Design and Simulation of Solenoid Valve Driving Circuit Module for High Pressure Common Rail Diesel Injector [S]. Applied Mechanics \& Materials, 2014, 602-605:2645-2648.

[2]. Chai Yunqiang. Research on Fuel Injection Characteristics of Electronically Controlled Fuel Injectors [J]. Energy and Conservation 2016, (03):72-75.

[3]. Fusin He, Tyesha Yang, Zhou in Xu, et al. Study on nonlinear injection of pulse width for injector injection characteristics of electronically controlled injector [J]. Journal of Henan University of Science and Technology, 2010, 31(5):1-2.

[4]. Guan Bing, Qian Yoyo, Yu Xiamen. Calibration and Test of Electronically Controlled Fuel Injectors [J]. Journal of Jilin University of Technology, 1996(4):15-19. 